Témoigner Témoigner. Entre histoire et mémoire

Getuigen Revue pluridisciplinaire de la Fondation Auschwitz

126 | 2018

Questions sur l'avenir du travail de mémoire

\title{
À lire / À voir / À suivre
}

\section{Q OpenEdition}

1 Journals

Édition électronique

URL : https://journals.openedition.org/temoigner/7646

DOI : 10.4000/temoigner.7646

ISSN : 2506-6390

\section{Éditeur :}

Éditions du Centre d'études et de documentation Mémoire d'Auschwitz, Éditions Kimé

\section{Édition imprimée}

Date de publication : 2 avril 2018

Pagination : 182-188

ISBN : 978-2-930953-06-9

ISSN : 2031-4183

Référence électronique

« À lire / À voir / À suivre », Témoigner. Entre histoire et mémoire [En ligne], 126 | 2018, mis en ligne le 24 janvier 2022, consulté le 04 février 2022. URL : http://journals.openedition.org/temoigner/7646 ; DOI : https://doi.org/10.4000/temoigner.7646 


\section{À lire/Ầ voir/Ā suivre}

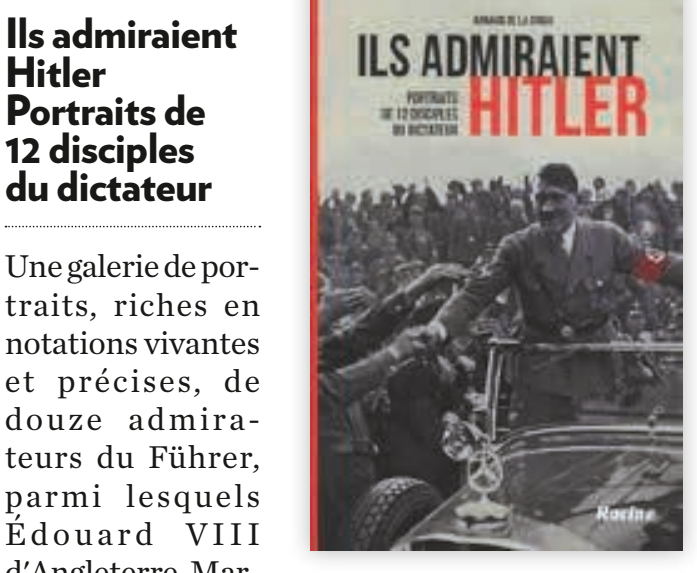

Histoire

économique

de.Vichy

hommes, les entreprises

Au regard des très nombreux livres portant sur la France pendant la Seconde Guerre économique et économique et sociale de la période d'Angleterre, Martin Heidegger, Charles Lindbergh, Amin al-Husseini, Knut Hamsun, Alois Hudal, Howard Phillips Lovecraft. Pourquoi ces douze persondifférents, célèbres chacune dans deur domaine, furent-elles fasciées par Hitler, un dictateur ées par Hitler, un dictateur qui ' prenait pour, le rédempteur de 'Allemác e' le sauveur de ThuVichy fait indéniablement figur de «parent pauvre »: les ouvrages inexistants. Pourtant, sujet son est sans doutecelio ce donain st sans dontecoluioullos connaisà de nombreux travaux de

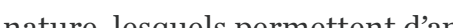
crent pes entrepris patronales, ou de comprendre les

conséquences des contraintes allemandes sur les échanges dans une France alors compartimentée en plus granter plus grand nombrécéséstoge éga-

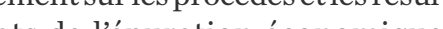
insi que sur les éveonomique, tages de láriode sur le mèrgu long terme. Il apparit au fin cung lenne. fuapparatt au final gur choix et/ou par force - le pays d'Eroix etou par force - le pays de l'effort de guerre allemand. $\Leftrightarrow$ Fabrice Grenard, Florent le Bot, Vichy Paris Perrin, 2017,500 pue

\section{La disparition de} Josef Mengele

1949 : Josef Mengele arrive en Argentine. Caché derrière divers pseudonymes, l'ancien médecin ortionnaire à Auschwitz croit pouvoir s'inventer une nires. Perón est bi lante, le monde entie lante, le monde entie vazis. Mais la traque La Disparition nazis. Mais la traque reprend et le médecin SS doit s'enfuir au Paraguay puis au Bresil. Son errance de déguisé et rongé pe, l'angoisse, nonge par langoisse, ne connaîtra plus de repit.. jusqu’à sa mort mysterieuse sur une plage
en 1979. Comment le médecin SS a-t-il pu passer entre les mailles du filet, trente ans durant? La Disparition de Josef Mengele est une plongé a Josef a dus du tars , vicilo Mengele en Amérique du Sud.

$\diamond$ Olivier Guez, La Disparition de Josef Mengele, Paris, Grasset, 2017

$\Leftrightarrow$ Recension de cet ouvrage dans Dotre numbero 127

Paul Halter, numéro 151610 D'un camp à l'autre

Ce livre de souvenirs à deux voix est dans le droit fil du devoir de perconpli avecla creation de la Fondatio Halter, est parler del'his de Paul . hor plus sombres de l'histodes les plú sonbres de listoire l'histoire du siecle et de parlor de austoire du siècle et de ceux qui, rer de tout se sont levés et ont dit non à l'oppression nazie. Paul "Halter numéro 151 610. D'un caul àl'autre décrit le parcoun exmpa linutre dect lo parco qui, même tionnel de cet honne qui, mêne en

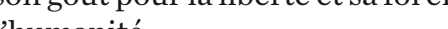

Ce qui ressort de la vie de ce rescapé des camps est l'extraordinaire cohicence de son destin, sa déter-

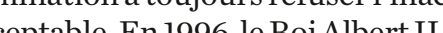
ceptable. En 1996, le RoiAlbert II anobli Pan halter. disprition en 2013, ce liveres permet de découvrir ce qu’a été

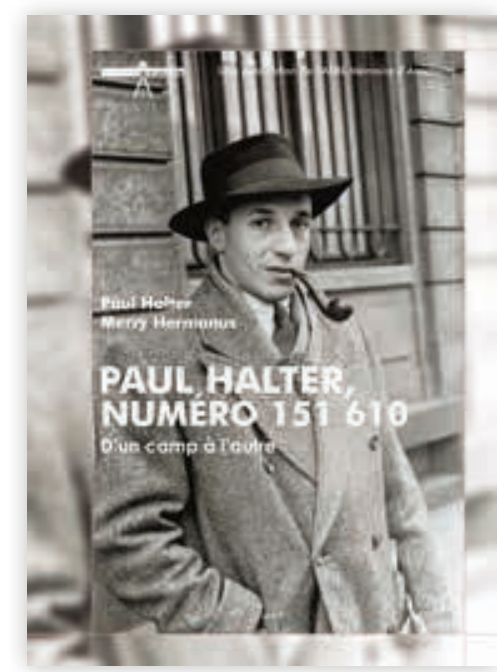

cette période à travers les souvenirs d'un homme dans son siècle. Paul Halter est né à Genève le 10 octobre 1920 et est décédé Molenbeek-Saint-Jean le 30 mars 2013. Il est devenu Belg par la naturalisation de ses parent au seront déportés via Drancy et début de l'O a Aupation Paul Halter s'enge da la nés Pant Halest a ế en 1943 et déporté de th arête en 1943 et deporté de Auschwitz. It est titulare de Ausch rifiques, en particulions honoexceptionnelle Croix d'Auschwitz e Pav Hater Mery Herasus $\diamond$ Paul Halter, Merry Hermanus, Paul Halter, numero 151 610. Dun campà $118 \mathrm{p}$.

$\rightarrow$ Recension de cet ouvrage dans

Retre numéro 127

\section{Krüger, un bourreau}

Qui était Friedrich-Wilhelm Krïer? Un officier allemand idéaliste qui entra à vingt ans dans la
Première Guerre mondiale. Un vat quicut le déshonneur $\mathrm{d}$ soldat qui vecut le déshonneú de tête de la Pologne occupée en 1939, au service d'Heinrich Himmler d'A discher jusqu’’ci le rôle majeur joú dus lextermination dedeur millions Juifs polonais. Le chef suprême de un poris. la police telass, quiftconstrúr les camps de Belzec, de Sobibor, de tiondughettodevarie a destruction delarésistarohestrise naise... Conste je jeune homme en deux décennies l'un des pires bourreaux de la Seconde Guere mondiale? Dans cette biographie . le Joun des archives allemandes, polodes archines allemandes, polole parcours nuancé d'un bourreau ordinaire sculpté par do bu violence, d'une guerre à 'autre.

$\rightarrow$ Nicolas Patin, Krüger, Un bourreau ordinaire, Paris, Fayard, « Biographies

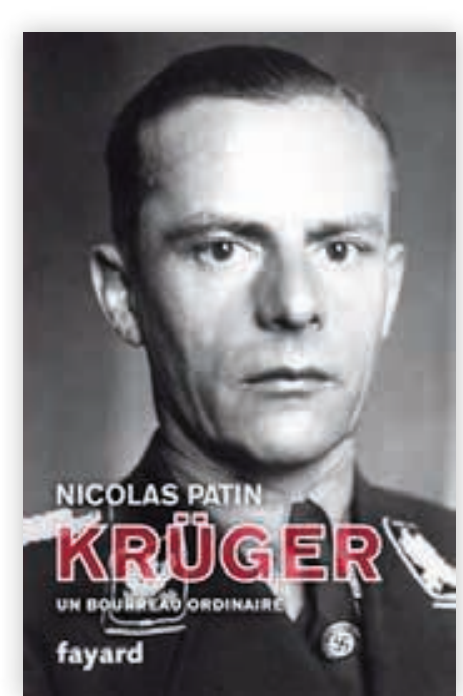


Éclairs

ointains

Percée à

Écrit pendant sa detention par un officier allemand Soviétiques, Éclairs Sointains retrace l'enfer du « chaudron de chaudron » de Stalind'homme - à la hauteur de tousces

soldats, officiers et sous-officier d'elstée Carl'evorís àlanéantissement. Car len

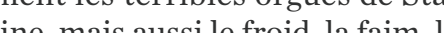
décrépitude physique, la daim, décrépitude physique, la démorahumn, a perte de tout sentiment d'être a la fois dat l'bis. force detre à la rois dans Thistoire mmédiate, celle de la politique treuses, et dans l'histoire de $l^{\prime} h$ mané : manité son objectif est bel et bien de dire que les hommes ne sont pas pours entro-tuer. Et ille fait avec pescite à fait pxeeptionnel.Desonexpérience de premiere main et des récits de ses compagnons de captivite, il tire une crudié de rudesset de tente de mêlées étondesse et de tendresse beautés d'une nature exposée aux beautés d'une nature exposée aux ravages des armes. Éclairs loinconfét ronan miraculé. Ilest coviétiques en 1949. Mais, intssoviétiques en 1949. Mais, incapabre de teur, une fois libéré, fait appel a un retrouver» son texte Illui faud plusieurs années pour le reudra Une version « abrégée » paraîtra en 1957. Cependant, l'histoire n'a
pas dit son dernier pas dit son dernier des années 1990 l'universitair universitaire Gansel retrouve le manuscrit origina danuscrit original dans les archives Gerlach arra vécu aux aléss de l'Histoire et s'impose désormais pose desormais comme un témoiurgence alors que la guerre fait hors du comm qui ron hors du conmun qui est bien Allemagne.

$\rightarrow$ Heinrich Gerlach, Éclairs lointains. Percée à Stalingrad, traduit de Gepner, Paris, Anne Carrière, 2017, 633 p.

Dans la nuit la

plus noire

se cache

'humanité

Récits des

Justes du

Jacques Roisin s'est rendu au Rwanda plusieurs années de suite afin de recueillir vingt Hutusquiont sauvé des Tutsis lors c de 1994 . Dans Au coeur du sauvetage, la première partie, l'auteur rapporte les têmoignages exhaustifs de six de procédé ? Pourquoi ont-ils sauvé alors que d'autres génocidaient? Étaient-ils écrasés par la peur? etc. Les six sauveteurs choisi pour cette première partie sont Zura, lensorceleuse crainte des danandas, qui a caché des Tutsis ciens venus pour tuer Gisimba ciens venus pour tuer, Gisimba, há elé pendant trois mois par les genouli livre lan orphelinat chés ; Rachid, l'imam qui a dirigé la lutte arién qui a dirigé des Tutsis de sa collined des Tutsis de sa colline de Mabare contre les attaques répétees des taire Hutu qui, de nuit, a le militaire Hutu qui, de nuit, a emmené pars lour dis versle Burundi; Edison, lex-genodes famillos de des fámilles dé Tutsis et organisé le rommerç sa fortune pour corromen sánocine pour corrompre les génocidaires et épargner ainsi les
Tutsis de sa col(intsis

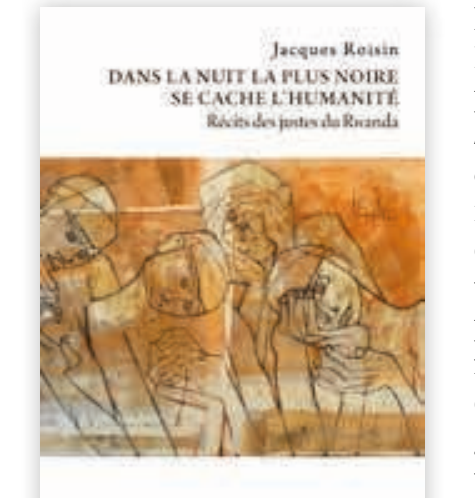

Dans la seconde partie, Les vrais héros sont silencieux, lauteurcommes vingt sauvedes vingt sauve-
teurs hutus. I teurs hutus. If présente les difgénocide rencon trées au rencontrees au Rwanda et rique de la fastosation et de la haine nti-Tutsis. À partir de nombreux extraits de témoignages, il interroge les motivations et les valeurs Tes

rwandaise mise en relatiórience ses consultations cliniques dans le domaine de la violence, il aborde une réflexion approfondie sur la question de la sollicitude humaine, autrement dit : comment le bien et le mal, comment l'humanité viennent-ils à lêtre humain?

$\diamond$ Jacques Roisin, Dans la nuit la plus noire se cache l'humanité. Récits des Justes du Rwanda, Bruxelles, Les $\diamond$ Recension de cet ouvrage dans

\section{Croire au matin \\ Cinq écrivains à la}

Palant rescapé d'Auschwitz

Qu'en est-il de l'enfance, de la transmission, de la parole, de lesponi, de lhumanité, soixantel'analyse historique etla politique ont échoué à faire ba politique ont échoué à faire barrage à la isme, ne à vate til las lactetion tismé, he reste-t-il pas la création défendre la liberté de pens pour, de défendre la liberté de penser, de viviet Chäret Elsabeth Brami, Mazarine Pingeot et Alice ZeniMazarine Pingeot et Alice ZeniCharles Palant, Charles Palant, íscapé des camps de la mort. A lheure où disparaissent les der(a) labarbarienazie, la littérature s'empare des quin'acessétout quin'acessétout au long de sa vie de nous exhorter à ne pas reurs auxquelles conduisent le racisme et l'antisé mitisme. Pour que nous, lecteurs, ions en mémoire l'extraordiaire témoignage d'un de ceux qui ont dit oui à la vie. À notre tour de e pas le laisser s'éteindre, de pnè à entendre aux génération futures.

$\triangle$ Elisabeth Brami, Noëlle Chätelet, Alexande Jardin, Mazarine Pingeo Chant rescapé d'Auschwitz Paris Calmann-Lévy, 2017, $160 \mathrm{p}$.

$\Leftrightarrow$ Recension de cet ouvrage dans notre numéro 127

\section{Lordre du jour}

L'Allemagne nazie a sa légende. On y voit un dontle triomphe parait inexorable. Mais si au fondement de ses premiers exploits se découvraient plutôt des marchandages, de vulgaires combinaisons d'intérêts Wes les glorieuses images de tehn un ime Panzers? Une simple panne! Un

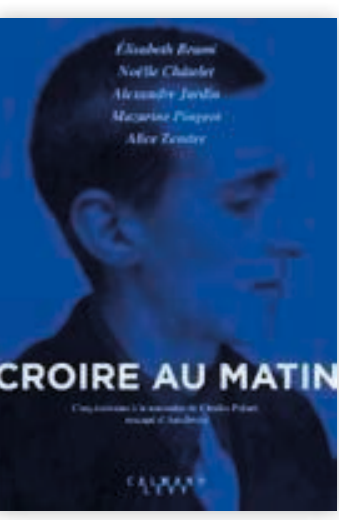

démonstration magistrale et grinante des coulisses de l'Anschlus parre et de 14 juillet.

$\Leftrightarrow$ Éric Vuillard, Loordre du jour, Arles, Actes Sud, 2017, $160 \mathrm{p}$

$\Leftrightarrow$ Recension de cet ouvrage dans

L’énigme Gerstein

La double vie improbable d'un acteur impliqué dans le système nazi qu'il dénonce parallèlement par tous les moyens. Depuis l'année 1933 Kurt Gerstein assiste avec effroi à la prise du pouvoir par Hitler en morales et ses convictions chrétiennes lions chrétiennes ner le régime bruta ner le régime brutal qui s'impose par le menraciste et la violence. de sa famille meurt subitement dans l'hôpital psychiatriqueoù il estinterné, victine de inistre Aktion T4 4 , . nant qu'il s'agit d'un crime d'Ét rant qu il s'agit dun crime d'Etat, Kurt Gerstein s'engage dans la SS pour enquêter de l'intérieur sur pour s'accomplit en secret dans la milice noire d'Hitler. Ce qu' découvrira dépasse de loin ce qu't imacinait Acteur directeme impliqué dans un système qu' dénonce daralèlement ma qu succès, Kurt Gerstein mènera une double viejusqu'àla fin de laguerre $\diamond$ Alain Le Ninèze, L'énigme Gerstein Paris, Ateliers Henry Dougier, 2017, 


\section{La Passeuse}

1942, quai de la gare des Aubrais : Bernard Prazan, 7 ans, serre fort la main de Thérèse Léopold qu'il doit appeler Tata mais qu'il connaît à peine. Quelques heures plus tôt, sa véritable tante les a confiés, lui et sa sœur, à cette inconnue pour qu'elle les fasse passer en zone libre. Mais au moment de quitter la gare, l'enfant comprend au regard delapasseuse qu'elle vales livrer auxAllemands. Pourtant, elle se ravise et les sauve. Dénoncée à son tour pour ce geste héroïque, elle sera déportée à Auschwitz-Birkenau, Mauthausen puis Ravensbrück. Elle en reviendra. De son vivant, Bernard a toujours affirmé à ses enfants qu'elle travaillait pour la Gestapo. Quiétaitelle vraiment? Une collabo repentie ou une Juste ignorée ? Pour connaître la vérité, Michaël Prazan s'est lancé dans la grande enquête de sa vie : celle de ses origines. Mêlant l'histoire de son père, enfant caché et homme taiseux, et celle de la passeuse qu'il a retrouvée et interrogée, il livre le récit bouleversant d'une famille persécutée et d'un sauvetage énigmatique.

$\rightarrow$ Michaël Prazan, La passeuse, Paris, Grasset, 2017, 320 p.

$\rightarrow$ Recension de cet ouvrage dans notre numéro 127

\section{Auschwitz est mon manteau}

La passeuse

Elle dit que « le tournesol est la fleur du Rom », qu'elle est une Tsigane qui aime « la pluie, le vent et l'éclair, quand les nuages masquent le ciel.» Elle dit qu'Auschwitz est son manteau et qu'elle ne connaît pas la peur car sa peur s'est arrêtée dans les camps. Elle dit que les notes de ses chansons en romani «sont toutes encore en désordre », mais que cela ne l'empêche pas de dire « Oui à la vie ». Elle, c'est Ceija Stojka, la première femme rom rescapée des camps de la mort à témoigner par l'art et par la poésie. Les poèmes de cette autodidacte ont été arrachés aux carnets où se mêlaient dessins, souvenirs de l'horreur, notes journalières et listes de mots allemands dont elle voulait apprendrel'orthographe. Publiés pour la première fois en France, ils révèlent une artiste majeure de notre temps.

$\rightarrow$ Stojka Ceija, Auschwitz est mon manteau. Et autres chants tsiganes,

Paris, Bruno

Doucey, 2018, 121 p.

\section{Rien où poser sa tête}

En 1921, Françoise Frenkel, une jeune femme passionnée par la langue et la culture françaises, fonde la première librairie française de Berlin, "La Maison du Livre ». Rien où poser sa tête raconte son itinéraire : contrainte en 1939 de fuir l'Allemagne, où il est devenu impossible de diffuser livres et journaux français, elle gagne la France, où elle espère trouver refuge. C'est en réalité une vie de fugitive qui l'attend, jusqu'à ce qu'elle réussisse à passer clandestinement la frontière suisse en 1943. Le récit, écrit en français, qu'elle en tire aussitôt dresse un portrait saisissant de la France du début des années quarante. De Paris à Nice, en passant par Avignon, Vichy, Grenoble, Annecy, Françoise Frenkel est témoin de la violence des rafles et vit sans cesse menacée en raison de ses origines juives. Tantôt dénoncée, tantôt secourue, incarcérée puis libérée, elle découvre des Français divisés par la guerre dont elle narre le quotidien avec objectivité. Rien où poser sa tête, soixante-dix ans après sa publication en 1945 à Genève, conserve,

Frangvioc Frenkel

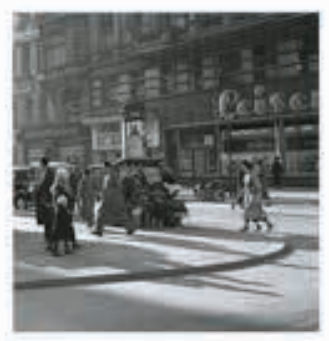

3

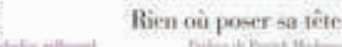
miraculeusement intacts, la voix, le regard, l'émotion d'une femme qui réussit à échapper à un destin tragique.

$\rightarrow$ Françoise

Frenkel, Patrice Modiano (préface), Rien où poser sa tête [1945], Paris, Gallimard, «Folio », 2018, 336 p. 